\title{
AN ILL-POSED PROBLEM FOR A STRICTLY HYPERBOLIC EQUATION IN TWO UNKNOWNS NEAR A CORNER ${ }^{1}$
}

\author{
BY STANLEY OSHER ${ }^{2}$
}

Communicated by Eugene Isaacson, December 4, 1973

In an earlier note [4] we gave a simple example of an ill-posed problem for a system of hyperbolic equations in a region whose boundary has a corner. The system was diagonal with coupling only at the boundary. Earlier we derived necessary and sufficient conditions for well-posedness [2] for a wide class of constant coefficient hyperbolic systems in such regions. In [3] we examined in some detail the phenomena which occur when these conditions are violated. The fundamental work for hyperbolic problems in regions with smooth boundaries was done by Kreiss [1].

It was pointed out by Sarason and Smoller [5] that the work of Strang [6] for the half-space problem implies that the corner problem is well posed for a strictly hyperbolic system in two unknowns iff the corresponding half-space problems are well posed. They constructed, using geometrical optics, a four dependent variable ill-posed example, where the half-space extensions were well posed.

In all the above-mentioned work, the boundary conditions imposed were local, i.e., of the form $B u=f$ at $x_{1}=0$, where $B$ is a matrix and $u$ is the unknown vector on the boundary.

We have noticed that much of the theory can be extended to nonlocal pseudo-differential boundary conditions. In particular, conditions of the form

$$
\boldsymbol{B}\left(w_{2}, \cdots, w_{n}, s\right) \hat{u}\left(0, w_{2}, \cdots, w_{n}, s\right)=\hat{f}\left(w_{2}, \cdots, w_{n}, s\right),
$$

where $B$ is a matrix-valued function of the dual variables $x_{i} \rightarrow w_{i}, t \rightarrow s$, can be treated. Such boundary conditions are reasonable when nonlinear problems are linearized. We shall discuss this in detail in a future paper.

Our purpose here is to show that for such boundary conditions wellposedness of the two half-space problems does not imply well-posedness of the corner problem, even in the strictly hyperbolic two unknown variable case.

AMS (MOS) subject classifications (1970). Primary 35L50, 35L30; Secondary 78A45.

Key words and phrases. Hyperbolic equation, well-posed, initial boundary.

${ }^{1}$ Research supported under N.S.F. grant number GP 29-273.

2 Fellow of the Alfred P. Sloan Foundation. 
We shall present necessary and sufficient algebraic conditions for wellposedness of this problem in the above-mentioned paper.

We consider the equation

$$
\left(\begin{array}{l}
u \\
v
\end{array}\right)_{t}=\left(\begin{array}{rr}
-1 & 0 \\
0 & 1
\end{array}\right)\left(\begin{array}{l}
u \\
v
\end{array}\right)_{x}+\left(\begin{array}{ll}
0 & 1 \\
1 & 0
\end{array}\right)\left(\begin{array}{l}
u \\
v
\end{array}\right)_{y}+\left(\begin{array}{l}
F_{1} \\
F_{2}
\end{array}\right)
$$

to be solved for the complex-valued functions $u$ and $v$ in the region $0<x$, $y, t$ with initial conditions

$$
u(x, y, 0)=v(x, y, 0)=0 .
$$

Next, apply a Laplace transform in $t$, use the initial conditions (2), and call the dual variable $s=\eta+i \xi$, with $n>0, \xi$ real. We have

$$
s \hat{U}-A \hat{U}_{x}-B \hat{U}_{y}=\hat{F},
$$

where $\hat{U}$ and $\hat{F}$ are the transformed 2 vectors $\left(\begin{array}{l}\hat{u} \\ \hat{v}\end{array}\right)$ and $\left(\begin{array}{l}\hat{F}_{1} \\ \hat{F}_{2}\end{array}\right)$, respectively; $A$ and $B$ are defined in (1).

We impose boundary conditions for $\eta>1$ :

$$
\begin{aligned}
& \text { (a) } \hat{u}(0, y, s)=-\frac{1+\sqrt{ }\left(1-c^{2}\right)}{c} \Phi_{1}(\xi) \hat{v}(0, y, s)+\hat{f}(y, s), \\
& \text { (b) } \hat{u}(x, 0, s)=-\frac{1+\sqrt{ }\left(1-c^{2}\right)}{c} \Phi_{2}(\xi) \hat{v}(x, 0, s)+\hat{g}(x, s),
\end{aligned}
$$

where $c$ is any real number, $0<c<1, \Phi_{1}(\xi), \Phi_{2}(\xi)$ are $C_{0}^{\infty}(-\infty, \infty)$ with $0 \leqq \Phi_{1} \leqq 1,-1 \leqq \Phi_{2} \leqq 1$, and $\Phi_{1}(\xi), \Phi_{2}(\xi) \equiv 1$ if $-\frac{1}{2}<\xi<\frac{1}{2}, \Phi_{1}(\xi) \equiv 0$, $\Phi_{2}(\xi) \equiv-1$ if $|\xi|>1$.

The standard estimate for problems of this type is

$$
\left(\eta-\eta_{0}\right)\|\hat{O}\|^{2}+\|\hat{O}\|_{B}^{2} \leqq K\left(\|\hat{F}\|^{2}+\|\hat{f}\|_{B_{1}}^{2}+\|\hat{g}\|_{B_{2}}^{2}\right)
$$

uniformly in $s=\eta+i \xi$, for $\eta>\eta_{0}, \eta_{0}>0$ and fixed.

The norms are defined as

$$
\|\hat{O}\|^{2}=\int_{0}^{\infty} \int_{0}^{\infty}\left(|\hat{u}(x, y, s)|^{2}+|\hat{v}(x, y, s)|\right)^{2} d x d y,
$$

$$
\|\hat{O}\|_{B}^{2}=\int_{0}^{\infty}\left[|\hat{u}(0, y, s)|^{2}+|\hat{v}(0, y, s)|^{2}+|\hat{u}(y, 0, s)|^{2}+|\hat{v}(y, 0, s)|^{2}\right] d y .
$$

\|\|$_{B_{1}}$ and \|\|$_{B_{2}}$ are defined analogously.

We have the following

THEOREM. No estimate of type (5) is possible for problem (3), (4)(a), (b). However, problem (3), (4)(a) in the region $0<x,-\infty<y<\infty$, and 
(3), (4)(b), in the region $-\infty<x<\infty, 0<y$ both obey estimates of type (5), where the norms are modified in an obvious fashion, to be integrals over half- rather than quarter-space.

Proof. For the quarter-space problem, we consider

$$
\hat{U}=\exp \left(-c s y-s x \sqrt{ }\left(1-c^{2}\right)\right)\left[\begin{array}{c}
1 \\
-c /\left(1+\sqrt{ }\left(1-c^{2}\right)\right)
\end{array}\right]
$$

for $s=\eta+i \xi, 0<\eta,-\frac{1}{2}<\xi<\frac{1}{2}$. This function satisfies the homogeneous equations (3), (4)(a), (b). Moreover, the norms on the left side of (5) are finite.

For the right half-plane problem we can easily obtain the estimate

$$
\left(\eta-\eta_{0}\right)\|\hat{U}\|^{2} \leqq K_{1}\left(\|\hat{F}\|^{2}+\|\hat{U}\|_{B}^{2}\right)
$$

independently of the boundary conditions (4)(a). (See e.g., Osher [2].)

We need only to obtain

$$
\|\hat{O}\|_{B}^{2} \leqq K_{2}\left(\|\hat{F}\|^{2}+\|\hat{f}\|_{B_{1}}^{2}+\|\hat{U}\|^{2}\right) .
$$

Moreover, in a standard fashion, we can assume $\hat{F} \equiv 0$. (See, e.g., Osher [2].)

We can solve equation (3) for $F \equiv 0$, with boundary conditions (4)(a). Fourier transform (3) in $y$, then multiply by $A^{-1}$. We have

$$
\tilde{U}_{x}-A^{-1}(s-B i w) \tilde{U}=0,
$$

where $w$ is the dual variable, $\tilde{U}=\mathscr{F} \hat{U}$,

$$
\text { (b) } \tilde{u}(0, w, s)=-\frac{1+\sqrt{ }\left(1-c^{2}\right)}{c} \Phi_{1}(\xi) \tilde{v}(0, w, s)+\tilde{f}(w, s) \text {. }
$$

Let

$$
\tilde{U}=T_{1}(w, s) \tilde{V}
$$

$T_{1}(w, s)$ is a unitary matrix-valued measurable function of $w, s$ such that

$$
T_{1}^{*}\left(A^{-1}(s-B i w)\right) T_{1}=\left(\begin{array}{cc}
-K_{+} & m_{12}(w, s) \\
0 & K_{+}
\end{array}\right)
$$

where $K_{+}=\sqrt{ }\left(s^{2}+w^{2}\right), \operatorname{Re} K_{+}>0$.

Thus, the general solution to (10)(a) which does not grow exponentially as $x \rightarrow+\infty$, is

$$
\tilde{V}=\left(\begin{array}{c}
\exp \left(-K_{+} x\right) \\
0
\end{array}\right) b_{1}(w, s)
$$


or

$$
\tilde{U}(0, w, s)=T_{1}(w, s)\left(\begin{array}{c}
b_{1}(w, s) \\
0
\end{array}\right)
$$

Apply the boundary condition (10)(b). Thus

$$
b_{1}(w, s)=\frac{f(w, s)\left[\left|s+K_{+}\right|^{2}+|w|^{2}\right]^{1 / 2}}{\left[\left(s+K_{+}\right)+\frac{1+\sqrt{ }\left(1-c^{2}\right)}{c} i w \Phi_{1}\right]} .
$$

It is easy to show that the quantity multiplying $f(w, s)$ is uniformly bounded in $w, s$, if $\eta>1$. Thus, this half-space problem is well posed.

We can do the analogous thing for the upper half-plane problem, arriving at

$$
\tilde{U}(w, 0, s)=T_{2}(w, s)\left(\begin{array}{c}
b_{2}(w, s) \\
0
\end{array}\right)
$$

Applying the boundary condition at $y=0$ leads to

$$
b_{2}(w, s)=\frac{\tilde{g}(w, s)(|s-i w|+|s+i w|)^{1 / 2}}{\left[\sqrt{ }(s-i w)-\frac{1+\sqrt{ }\left(1-c^{2}\right)}{c} \sqrt{ }(s+i w) \Phi_{2}(\xi)\right]},
$$

where each square root has positive real part Again we have $\left|b_{2}(w, s)\right| \leqq$ $K_{3}|\tilde{g}(w, s)|$ if $\eta>1$. Thus the half-space problem is well posed. Q.E.D.

\section{BIBLIOGRAPHY}

1. H.-O. Kreiss, Initial boundary value problems for hyperbolic systems, Comm. Pure Appl. Math. 23 (1970), 277-298.

2. S. Osher, Initial boundary value problems for hyperbolic systems in regions with corners. I, Trans. Amer. Math. Soc. 176 (1973), 141-164.

3. - Initial boundary value problems for hyperbolic systems in regions with corners, II, Trans. Amer. Math. Soc. (to appear).

4. - An ill-posed problem for a hyperbolic equation near a corner, Bull. Amer. Math. Soc. 79 (1973), 143-144.

5. L. Sarason and J. Smoller, Geometrical optics and the corner problem, Arch Rational Mech. Anal. (to appear).

6. G. Strang, Hyperbolic initial-boundary value problems in two unknowns, J. Differential Equations 6 (1969), 161-171. MR 39 \#633.

Department of Mathematics, State University of New York at Stony Brook, STONY BrOOK, New YORK 11790 